\title{
Commentary on Fontaine et al.: Safety and efficacy of deep brain stimulation in refractory cluster headache: a randomized placebo- controlled double-blind trial followed by a 1-year open extension
}

\author{
Anna Ambrosini · Jean Schoenen
}

Received: 1 December 2009/Accepted: 14 December 2009/Published online: 5 January 2010

(C) Springer-Verlag 2009

Among primary headaches, cluster headache is certainly the most painful and unbearable, particularly for the patients suffering from its chronic form. It is characterized by attacks of unilateral periorbital, frontal and/or temporal pain associated with ipsilateral autonomic signs (ptosis, miosis, conjunctival injection, tearing, rhinorrhoea, nasal congestion). Its episodic form is characterized by bouts (clusters) of weeks or months, separated by headache-free intervals of variable length (months or years), but in chronic cluster headache $(\mathrm{CCH})$, which affects almost $10 \%$ of patients, the attacks persist for at least 1 year without remission, or with remissions lasting less than 1 month [1]. These patients often require one or more preventive drugs for relief, such as steroids, verapamil, lithium carbonate and methysergide. Unfortunately, about $1 \%$ of $\mathrm{CCH}$ patients become refractory to all existing pharmacological treatments.

In fact, suffering from intractable $\mathrm{CCH}$ is an atrocious condition, which affects every aspect of the patients' lives, and may even push some of them to commit suicide as the ultimate desperate solution to avoid pain.

Thus, it is imperative for headache clinicians and researchers, to test any possible remedy to this condition by invasive and non-invasive surgical procedures when pharmacological remedies have failed.

Over the years, many such procedures have been used, such as radiofrequency lesions, glycerol injections or balloon compressions of the gasserian ganglion, gamma knife

\footnotetext{
A. Ambrosini $(\square)$

Headache Clinic, INM Neuromed, IRCCS,

Pozzilli (Isernia), Italy

e-mail: anna.ambrosini@neuromed.it

J. Schoenen

Headache Research Unit, Department of Neurology,

University of Liège, Liège, Belgium
}

surgery or root section of the trigeminal nerve, trigeminal tractotomy, lesions of the nervus intermedius or greater superficial petrosal nerve, blockade or radiofrequency lesions of the pterygopalatine ganglion, and microvascular decompression of the trigeminal nerve combined with nervus intermedius section [2]; none of these have displayed satisfactory long-term results.

In addition, when neuromodulation methods were first applied with success in some cases of intractable $\mathrm{CCH}$ [3], they immediately had a great resonance in the scientific community and aroused great hope in affected patients. The neuromodulation techniques used up to now are deep brain stimulation (DBS) of the ventro-posterior hypothalamus [3-6] and occipital nerve stimulation [7, 8]. Observational studies of hypothalamic DBS (hBDS) seemed to report slightly better results, but also more serious side effects, culminating in fatal cerebral haemorrhage in one patient. Because of this potential risk of hDBS procedures, it is of uttermost importance to carefully select $\mathrm{CCH}$ patients to whom hDBS will be proposed and to provide evidence-based proof of its clinical efficacy.

Criteria defining drug-resistant chronic cluster headache (iCCH) patients are based on experts' opinion and widely accepted $[9,10]$. The consensus is that patients cannot be considered drug-resistant unless drugs known to be effective in cluster headache and belonging to at least three different pharmacological classes have been administered at a sufficient dose and for a sufficient time.

In two studies [5, 6], attack recurrence at varying delays was described when the stimulator was switched off or when the battery ran flat, suggesting that the clinical effect was probably not due to the natural history of the disorder. Since the stimulation is not perceived by the patient, these observations also do not favour a placebo effect, although they cannot rule it out [11]. A placebo-controlled study was 
thus urgently needed, and Fontaine et al. deserve praise for organizing and completing such a trial embracing a substantial number of patients [12].

The study by Fontaine et al., the first controlled trial for hDBS in $\mathrm{CCH}$, was a multicenter double-blind study conducted on 11 patients, who after the electrode implantation were randomized to a "switch on" or a "switch off" condition [12]. This "randomized phase" consisted in a cross-over trial, where in the first month, a group of patients received effective stimulation and another group "sham" stimulation, whereas in the second month the stimulators of the first group were switched off and those of the second group switched on. These two periods were separated by a week of "wash-out". The randomized phase was followed by a 1-year open phase, during which every patients received effective hypothalamic stimulation.

In the randomized phase, no significant effect was found for $\mathrm{hDBS}$ in comparison with sham stimulation, whereas the open phase confirmed the good results obtained in previous open-labelled studies, i.e. $\geq 50 \%$ reduction of attack frequency in about $60 \%$ of patients.

The data obtained from the randomized phase, therefore, provide no evidence favouring the use of $\mathrm{hDBS}$ in drugresistant $\mathrm{CCH}$ patients, which contrasts with the longer open-label phase and previous studies. This discrepancy may have several explanations. The most relevant one is probably the rather short, 1-month, duration of the treatment periods in the randomized phase. Hypothalamic DBS, like occipital neurostimulation, is likely to act by slow neuromodulatory processes which take time to have an effect. Although in previous studies some patients had rapid improvement after hDBS, significant beneficial effect was delayed in many others, and it is the rule in occipital nerve stimulation. This may explain why patients who did not respond in the randomized phase of the trial became responders in the subsequent open-label phase after a longer duration of stimulation. Unfortunately, the authors were not able to provide stronger evidence for the beneficial effect in the open phase by switching the stimulators off, since the patients to whom a second longer randomized phase was proposed did not accept the risk of being allocated to the "off" arm and losing the treatment effect. On the other hand, the patients who were implanted in Fontaine et al.'s study are not totally comparable to those included in most open studies. In the latter, intractability was defined on the basis of stricter criteria including treatment failure to at least four different classes of preventive drugs [9], while in this controlled trial patients were included if they had failed (or not tolerated) only two medications, verapamil and lithium. Admittedly, less refractory patients might in theory respond better to hypothalamic stimulation, which would have increased the likelihood of a favourable outcome, and intractability may not be irreversible as found in another study [6] where even patients considered to be intractable went into remission while on a waiting list for DBS. The problem is merely an ethical one, as the international consensus is that hDBS, which is not a riskless procedure, should be restricted to patients who resist to all available non-invasive preventive treatments.

In conclusion, this first randomized controlled study of hDBS unfortunately fails to provide level I evidence for this procedure in drug-resistant chronic cluster headache, as no significant effect was found during the randomized 1-month phase. Thanks to the good efficacy found in the open-label phase, however, it supports the favourable results from previous observational studies and confirms that most $(60 \%)$, though not all, patients can be significantly improved by the procedure.

Conflict of interest None.

\section{References}

1. Headache Classification Subcommittee of the International Headache Society (2004) The international classification of headache disorders, 2nd edn. Cephalalgia 24(Suppl 1)

2. Matharu MS, Boes CJ, Goadsby PJ (2003) Management of trigeminal autonomic cephalgias and hemicrania continua. Drugs 63(16): $1637-1677$

3. Leone M, Franzini A, Bussone G (2001) Stereotactic stimulation of posterior hypothalamic gray matter in a patient with intractable cluster headache. N Engl J Med 345(19):1428-1429

4. Leone M, Franzini A, Broggi G, May A, Bussone G (2004) Longterm follow-up of bilateral hypothalamic stimulation for intractable cluster headache. Brain 127(10):2259-2264

5. Leone M, Franzini A, Broggi G, Bussone G (2006) Hypothalamic stimulation for intractable cluster headache: long-term experience. Neurology 67(1):150-152

6. Schoenen J, Di Clemente L, Vandenheede M, Fumal A, De Pasqua V, Mouchamps M, Remacle JM, de Noordhout AM (2005) Hypothalamic stimulation in chronic cluster headache: a pilot study of efficacy and mode of action. Brain 128:940-947

7. Burns B, Watkins L, Goadsby PJ (2007) Treatment of medically intractable cluster headache by occipital nerve stimulation: longterm follow-up of eight patients. Lancet 369(9567):1099-1106

8. Magis D, Allena M, Bolla M, De Pasqua V, Remacle JM, Schoenen J (2007) Occipital nerve stimulation for drug-resistant chronic cluster headache: a prospective pilot study. Lancet Neurol 6(4):314-321

9. Leone M, May A, Franzini A, Broggi G, Dodick D, Rapoport A, Goadsby PJ, Schoenen J, Bonavita V, Bussone G (2004) Deep brain stimulation for intractable chronic cluster headache: proposals for patient selection. Cephalalgia 24(11):934-937

10. Goadsby PJ, Schoenen J, Ferrari MD, Silberstein SD, Dodick D (2006) Towards a definition of intractable headache for use in clinical practice and trials. Cephalalgia 26:1168-1170

11. Nilsson Remahl AI, Laudon ME, Cordonnier C, Goadsby PJ (2003) Placebo response in cluster headache trials: a review. Cephalalgia 23(7):504-510

12. Fontaine D, Lazorthes Y, Mertens P, Blond S, Géraud G, Fabre N, Navez M, Lucas C, Dubois F, Gonfrier S, Paquis P, LantériMinet M (2010) Safety and efficacy of deep brain stimulation in refractory cluster headache: a randomized placebo-controlled double-blind trial followed by a 1-year open extension. J Headache Pain 10. doi:10.1007/s10194-009-0169-4 